\title{
Design of Rehabilitation Training System for Pelvic Floor Dysfunction
}

\author{
Yongfeng Liu ${ }^{1}$, Chunna $\mathrm{Li}^{2}$, Jun Zhong ${ }^{1 *}$, Liming Cai1 and Kai Guo \\ ${ }^{1}$ Suzhou Institute of Biomedical Engineering and Technology, Chinese Academy of Science, Suzhou, \\ Jiangsu, 215163, China. \\ 2964 Hospital of the Joint Service Support Force of the Chinese People's Liberation Army, \\ Changchun 130062, China.
}

\begin{abstract}
Pelvic floor dysfunction has caused pain to the lives of the majority of female patients, and repeated visits to the hospital for treatment have also caused inconvenience to patients. Therefore, a portable pelvic floor dysfunction rehabilitation training system is designed to help female patients with personalized and private treatment. The pelvic floor dysfunction rehabilitation system realizes the independent training mode selection through the APP client, assists the pelvic floor muscle rehabilitation training through the electrical stimulation module, and can realize the recovery situation of the pelvic floor muscle strength in real time through the $\mathrm{AD}$ acquisition module. The experimental results show that the pelvic floor dysfunction rehabilitation training system implements bipolar electrical stimulation pulses, and the EMG signals collected at the same time clearly show the EMG signal strength and strength maintenance time.
\end{abstract}

\section{Introduction}

Childbirth and pregnancy activities, abdominal surgery, etc [1-3], will cause varying degrees of pelvic floor function reduction or obstacles, which may be accompanied by lower abdominal discomfort, urinary incontinence, abnormal urination, abnormal bowel movements, abnormal vaginal secretions, etc., which will bring female patients later life Inconvenience and pain, taking pelvic floor muscle rehabilitation training in time for childbirth or after surgery is of great benefit in preventing postpartum complications and restoring pelvic floor muscle function $[4,5]$.

At present, many studies have shown that pelvic floor electrical stimulation has obvious effects on pelvic floor muscle rehabilitation [6-8], but due to the large hospital treatment equipment and long treatment cycle, it is not convenient for female patients to complete the treatment course better. In order to enable female patients to carry out early and timely intervention on pelvic floor dysfunction at home, it is designed a rehabilitation training system for pelvic floor dysfunction.

The pelvic floor dysfunction rehabilitation training system designed in this paper uses the constant current electrical stimulation module of the pelvic floor dysfunction rehabilitation

\footnotetext{
*Corresponding author: zhongj@sibet.ac.cn
} 
training device to electrically stimulate the pelvic floor muscles, and uses the $\mathrm{AD}$ module to collect pelvic floor muscle strength signals and send the EMG signals through Bluetooth Go to the mobile APP for display and management. The patient uses the APP to view the current pelvic floor muscle signal strength and pelvic floor muscle recovery. This pelvic floor dysfunction rehabilitation training system has the advantages of high integration, low power consumption, high measurement accuracy, etc., which is convenient for female patients to use and relieve the pain caused by pelvic floor dysfunction.

\section{Overall System Design}

The pelvic floor dysfunction rehabilitation training system includes pelvic floor dysfunction rehabilitation training apparatus and APP. The pelvic floor dysfunction rehabilitation training device includes a main control MCU module, a constant current electrical stimulation module, an $\mathrm{AD}$ acquisition module, a Bluetooth module and a power management module, and the APP part displays and manages training data. The functional block diagram of this system is shown in figure 1 .

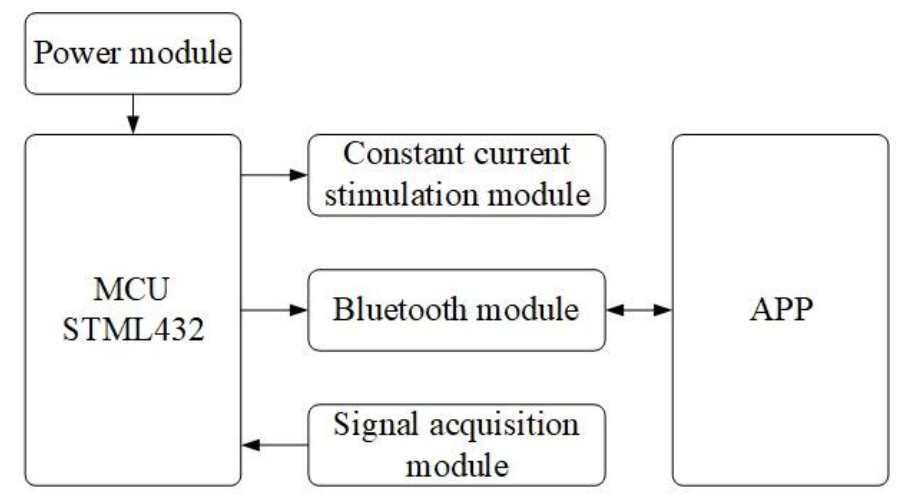

Fig. 1. System block diagram.

\section{Selection of Main Components}

\subsection{Main Control Chip STM32L432}

STM32L432 is an ultra-low power microcontroller based on 32-bit RISC core, and its operating frequency can reach $80 \mathrm{MHz}$. The Cortex-M4 core has a floating-point arithmetic unit that supports all Arm ${ }^{\circledR}$ single-precision data processing instructions and data types. This chip provides a 12 -bit $\mathrm{ADC}$, the speed can reach $5 \mathrm{Msps}$, while also providing low-power $\mathrm{RTC}$ and timer to reduce power consumption.

\subsection{Bluetooth Chip NRF51822}

nRF51822 is a powerful and highly flexible multi-protocol integrated circuit chip, commonly used in $2.4 \mathrm{GHz}$ Bluetooth low energy applications. nRF51822 has 256kBFlash and $16 \mathrm{kBRAM}$, and also has a wealth of analog and digital peripheral products. It can interact through the programmable peripheral product interconnection (PPI) system without CPU involvement, and can flexibly design the $\mathrm{PCB}$ through the pin mapping scheme. It is now used in medical, fitness, consumer electronics and many other fields. 


\subsection{AD Acquisition Chip ADS1292}

ADS1292 is a multi-channel 24-bit delta-sigma analog-to-digital converter with built-in programmable gain amplifier, internal reference and an on-board oscillator. Its operating data rate is up to $8 \mathrm{KSPS}$, and $335 \mathrm{uW}$ per channel in low power mode.

\section{System Hardware Design}

\subsection{Constant Current Electrical Stimulation Module}

The constant current electrical stimulation module consists of a booster circuit and an $\mathrm{H}-$ bridge circuit, as shown in figure 2. According to the set stimulation current value, STIM_PWM1 transmits a $30 \mathrm{KHz}$ frequency PWM wave, and the duty ratio corresponds to the set current value. Through the charge pump circuit composed of L3, D2 and Q5, the input $3.3 \mathrm{~V}$ is pumped to a high voltage $(10-55 \mathrm{~V})$ and rectified by $\mathrm{C} 28$. Q6 is designed as a protection circuit. When the detection point STIM_V2 detects that the pump voltage exceeds the target voltage, the circuit is discharged through Q6. At this point, the circuit completes the boosting process and boosts the 3.3 V voltage to a constant voltage STIM_VMAX output.

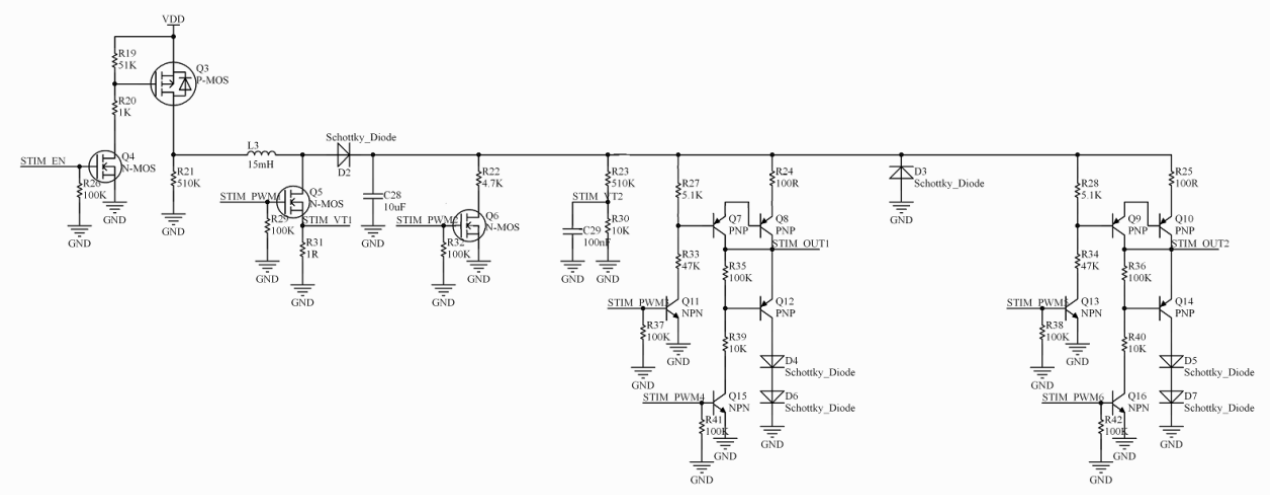

Fig. 2. Circuit diagram of constant current electrical stimulation.

The stimulation pulse waveform generated by the H-bridge circuit in the system is a bipolar pulse. The positive and negative pulse charges are equal. The positive and negative charges neutralize each other in a cycle, which can reduce the accumulation of charges in the skin tissue, which is not easy to cause muscle fatigue and can be avoided by Unipolar pulse stimulation causes problems such as a tingling sensation due to charge accumulation. When the system is working, a constant current circuit is formed by Q7, Q8, Q11, Q15, R27, R33, $\mathrm{R} 35, \mathrm{R} 39$, and the second half is composed of another constant current circuit, and positive and negative stimulation is performed through the form of H bridge: STIM_PWM3 Flat, STIM_PWM4 low level, STIM_PWM5 low level, STIM_PWM6 high level, at this time STIM_OUT1 level is higher than STIM_OUT2, positive input; when the control level is all reverse, the corresponding input is negative.

\subsection{AD Acquisition Module}

ADS1292 selects 1-channel differential signal input, and after 24-bit analog-to-digital conversion, data transmission is performed through the SPI interface of the MCU. 


\subsection{Bluetooth Data Transmission Module}

The serial port of NRF51822 is connected to the serial port of MCU. The APP on the mobile phone sends the training mode command via Bluetooth. The data processed by the MCU is sent to the Bluetooth module through the serial port, and then sent to the APP on the mobile phone via Bluetooth for display and management.

\section{System Software Design}

\subsection{Control Software Design of Rehabilitation Training Apparatus for Pelvic Floor Dysfunction}

The control program of the pelvic floor dysfunction rehabilitation training instrument includes two parts: the main control program based on STM32L432 and the Bluetooth data upload program based on NRF51822. Keil uVision5 is used to write STM32 embedded program and Bluetooth data upload program. The entire drive control flow of STM32 is shown in figure 3. After the system is turned on, initialize the timer, SPI, PWM, serial port, etc., wait for the mobile phone APP to send training instructions via Bluetooth, and after receiving the instructions, set the frequency, pulse width and other parameters of the constant current electrical stimulation module according to the mode selected by the instruction, and Start the electrical stimulation training, and at the same time use the AD acquisition module to collect pelvic floor muscle strength signals. STM32 sends the EMG signal data to the Bluetooth module NRF51822 through the serial port. After receiving the data, the Bluetooth module NRF51822 sends it to the mobile phone APP via Bluetooth for display and management.

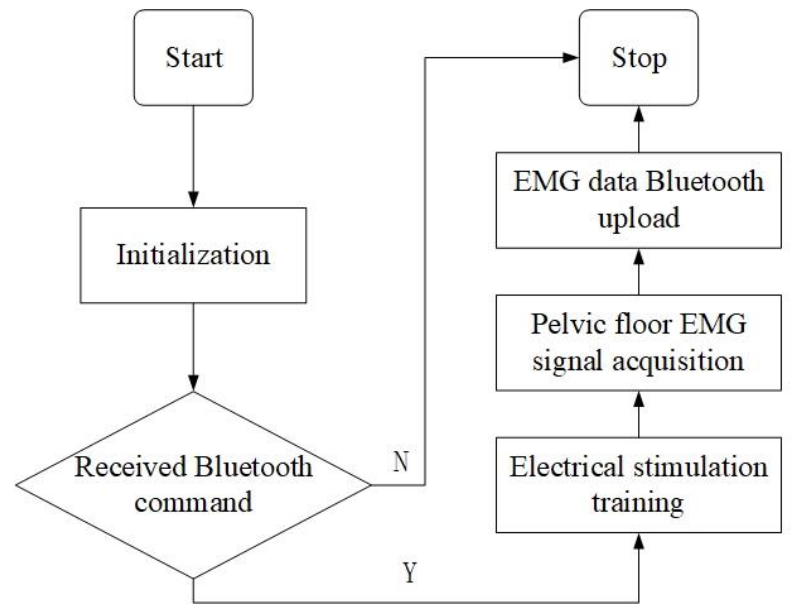

Fig. 3. Embedded drive control flow chart.

\subsection{APP Design of Pelvic Floor Dysfunction Rehabilitation Training System}

The pelvic floor dysfunction rehabilitation training system APP provides patients with the choice of treatment mode. At the same time, the pelvic floor dysfunction rehabilitation training device uploads the pelvic floor myoelectric signal to the mobile phone APP for display. The patient can view the strength of the pelvic floor muscle strength signal through 
the APP By comparing with historical data, you can view the recovery trend, so that patients have a clearer understanding of their pelvic floor function recovery.

\section{Experimental Test}

\subsection{Simulated Electrical Stimulation Experiment}

In the experiment, a $1 \mathrm{~K} \Omega$ resistor was used to simulate the human body impedance. The output of the electrical stimulation module was connected to both ends of the resistor through the electrode pads. The electrical stimulation parameters were set for electrical stimulation output. The actual waveform was observed through an oscilloscope. The experimental results are shown in figure 4. From the experimental results, it can be seen that the output waveform amplitude is $16 \mathrm{~V}$, the stimulation frequency is $35 \mathrm{~Hz}$, and the pulse width is $125 \mathrm{uS}$, which meets the design requirements of electrical stimulation therapy.

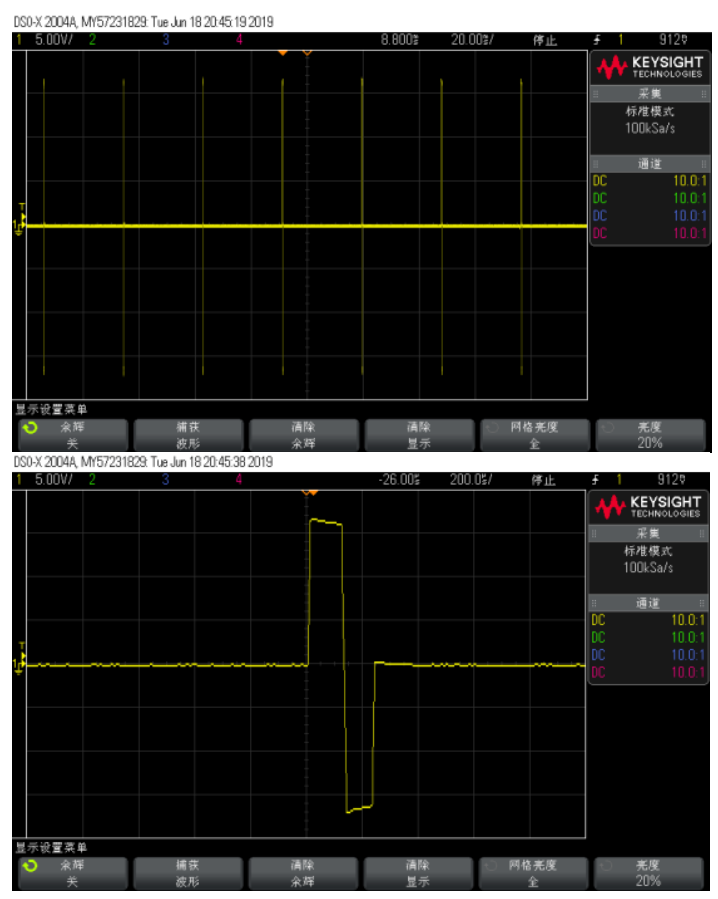

Fig. 4. Electrical stimulation signal diagram.

\subsection{EMG Signal Acquisition Experiment}

In the experiment, the electromyographic signal of the human biceps was connected to the input terminal of the $\mathrm{AD}$ acquisition module through the electrode sheet, and the $5 \mathrm{~kg}$ weight was lifted by the arm every 5 seconds to generate the electromyographic signal of the biceps, and then the weight was dropped for 5 seconds Repeat the test for 1 minute, the test results are shown in figure 5. It can be clearly seen from the figure that the muscle EMG signal becomes stronger when the muscle is exerted, and during the time period of maintaining the strength, the EMG signal is basically maintained at about $100 \mu \mathrm{V}-150 \mu \mathrm{V}$. The strength and 
duration of EMG signals are both important indicators for evaluating the recovery of pelvic floor muscle strength.

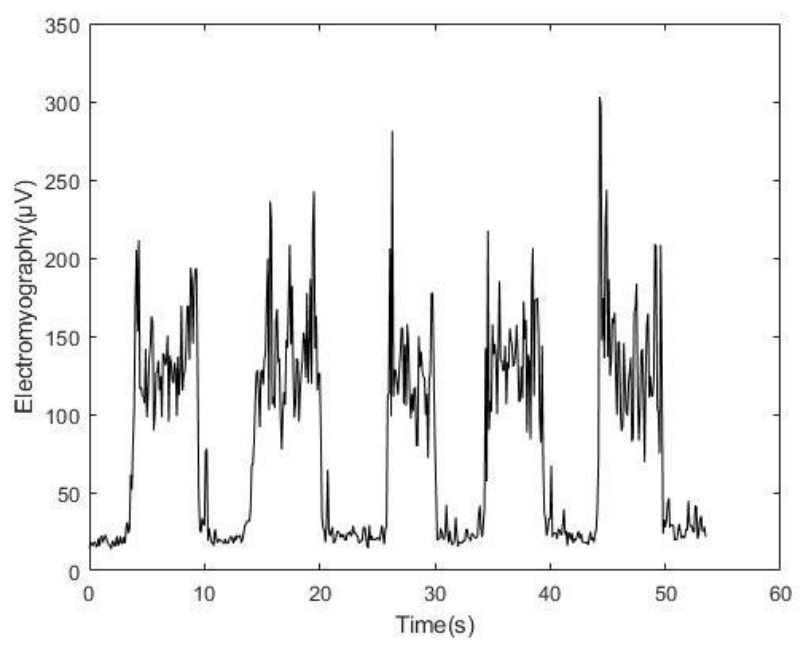

Fig. 5. EMG signal acquisition diagram.

\section{Conclusion}

The pelvic floor dysfunction rehabilitation training system includes pelvic floor dysfunction rehabilitation training instrument and APP client. It has the functions of large-scale hospital equipment and is also miniaturized and portable, not limited by time and space. It is used in various occasions and has three major functions: electrical stimulation, muscle strength test and biofeedback autonomous training. Induce the electrical activity of the pelvic floor muscles of the patient through the vaginal probe to evaluate the strength of the pelvic floor muscles, and then start the electrical stimulation program to stimulate the pelvic floor muscles and their inner nerves with electric current to directly induce a therapeutic response, which can be a good solution for women Patients with pelvic floor dysfunction $[9,10]$.

\section{Acknowledgements}

This work was supported by "Jiangsu Science and Technology Plan: Construction and Research of Rehabilitation Treatment and Information Management Platform for Pelvic Floor Dysfunction" (BE2017672).

\section{References}

1. W Y Du, Y F Zhou, Y T Yuan, etc. The effect of Kegel exercise combined with pelvic floor rehabilitation on pelvic floor function in patients undergoing laparoscopic extrafascial hysterectomy, Journal of Navy Medicine, 42 (2) 203-205(2020)

2. F F Jia, Analysis of the effect of postpartum pelvic floor rehabilitation exercise combined with bioelectric stimulation on the postpartum pelvic floor function rehabilitation effect and prognosis, Modern Chinese Medicine Application, 14 (7) 226227 (2020) 
3. LM Yuan, L Li, D X Liu, etc. Rehabilitation effects of pelvic floor muscle training on female pelvic floor dysfunction diseases, Modern Medicine and Health, 36, 6: 918-919 (2020)

4. I MILSOM, Can we predict and prevent pelvic floor dys-function? Int Urogynecol J, 26(12) 1719-1723 (2015)

5. K JUNDT, U PESCHERS, H KENTENICH, The investigation and treatment of female pelvic floor dysfunction, Dtsch Arztebl Int,112(33/34) 564-574 (2015)

6. L S Liu, Application of pelvic floor muscle training and electrical stimulation in postpartum pelvic floor muscle rehabilitation, Journal of Mathematical Medicine, 33(4) 606-607 (2020)

7. $\mathrm{CH}$ Wang, Pelvic floor rehabilitation training physiotherapy instrument in patients with pelvic floor organ prolapse application effect. Medical Equipment, 33(6) 153-155 (2020)

8. T R Wang, X Zhou, L J Jiang, The effect of comprehensive treatment of pelvic floor and its influence on quality of life of urinary incontinence in the elderly women. Chin $\mathrm{J}$ Clin Healthc, 23(2) 252-255 (2020)

9. S J MADILL, S P DRILET S, A TANG A, etc. Changes in urethral sphincter size following rehabilitation in older women with stress urinary incontinence, Int Urogynecol J, 26(2) 277-283 (2015)

10. A RADZIMINSKA, A STRACZYNSKA, M W RAJEK, etc, The impact of pelvic floor muscle training on the quality of life of women with urinary incontinence: a systematic literature review. ClinInterv Aging, 13(5) 957-965 (2018) 